\title{
AUTOMATIZACIÓN Y MONITORIZACIÓN DE UNA INSTALACIÓN DE ENSAYO DE MOTORES
}

\author{
Miguel Ochoa Vega \\ AVL Ibérica S.A. Departamento de Ingeniería eléctrica y automática. \\ Paseo Arco de Ladrillo 68, planta 5a , Valladolid, 47007, España. \{miguel.ochoa@avl.com $\}$ \\ Eduardo Julio Moya de la Torre, Fco. Javier García Ruiz, Alfonso Valentín Poncela Méndez \\ Instituto de las Tecnologías Avanzadas de la Producción, ITAP. \\ Escuela de Ingenierías Industriales, Paseo del Cauce 59, Valladolid, 47011, España. \{poncela@eii.uva.es\}
}

\section{Resumen}

El objetivo del presente artículo es el de presentar la automatización y monitorizado (local y remoto) de las instalaciones (facilites) de un edificio de celdas de ensayo para probar motores de tractor en Turquía. El edificio de celdas de ensayo tiene la peculiaridad de haber sido prefabricado en contendedores en Austria y trasladado hasta su ubicación final.

Las tareas aquí presentadas describen los diferentes componentes del sistema de control (PLC's), los interface hombre máquina (HMI's), los medios para acceso remoto, y las comunicaciones entre dispositivos. Se trata de una instalación real, estable $y$ en funcionamiento.

Palabras Clave: contenedor banco de ensayo, ensayo de motores, automatización, monitorizado, comunicaciones industriales, PLC, SCADA, HMI, VPN, acceso remoto.

\section{INTRODUCCIÓN}

El trabajo, a nivel industrial, queda enmarcado en el campo de la industria de automoción. Concretamente dentro del desarrollo y pruebas de motores para maquinaria agrícola, tractores para cultivo. En esta industria, los requisitos de plazo del mercado son cada vez más demandantes. Nos encontramos con que los tiempos de desarrollo de un vehículo se han visto disminuidos drásticamente y las previsiones de futuros son aún más exigentes, como se puede ver en la Figura 1.

El tiempo de desarrollo a principios de 2012 era de unos 42 meses. Las previsiones [1] muestran que en 2020 este tiempo será de únicamente 20 meses. Si nos centramos en el mercado de maquinaria agrícola, al requerimiento de plazo se debe sumar el aumento de la demanda de maquinaria. La producción de cereal ha aumentado en un $40 \%$ desde principios de este siglo [2].

\section{0\% REDUCTION OF DEVELOP LEAD-TIME UNTIL 2020}

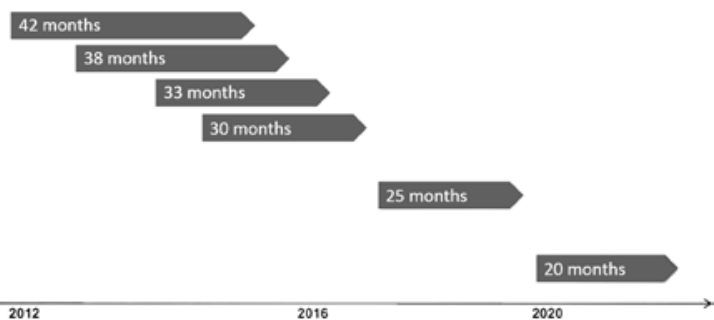

Figura 1: Previsiones desarrollo automóvil. SAE. Morey, 2014

Queda claro que hay que probar más vehículos y hay que probarlos en menos tiempo. A mayores, el cómo hay que probarlos también juega en esta ecuación. Las pruebas más limitantes a día de hoy son las de emisiones contaminantes. Las normativas EURO, conocidas en el ámbito de aplicación de los turismos, tienen su equivalente en la norma "Reglamento (UE) 2016/1628 del Parlamento Europeo y del Consejo del 14 de septiembre 2016 [4]. Véase la Tabla 1sobre los requisitos relativos a los límites de emisiones de gases y partículas contaminantes y a la homologación de tipo para los motores de combustión interna que se instalen en las máquinas móviles no de carretera.

Tabla 1: Límites de emisiones maquinaria

\begin{tabular}{|c|c|c|c|c|c|c|c|c|}
\hline $\begin{array}{c}\text { Fase de } \\
\text { emisiones }\end{array}$ & $\begin{array}{c}\text { Subcategoría } \\
\text { motor }\end{array}$ & $\begin{array}{c}\text { Potencia } \\
\mathbf{k W}\end{array}$ & $\begin{array}{c}\mathbf{C O} \\
\mathbf{g} / \mathbf{k W h}\end{array}$ & $\begin{array}{c}\mathbf{H C} \\
\mathbf{g} / \mathbf{k W h}\end{array}$ & $\begin{array}{c}\mathbf{N O x} \\
\mathbf{g} / \mathbf{k W h}\end{array}$ & $\begin{array}{c}\mathbf{P M} \\
\mathbf{g} / \mathbf{k W h}\end{array}$ & $\begin{array}{c}\mathbf{P N} \\
\# / \mathbf{k W h}\end{array}$ & $\mathbf{A}$ \\
\hline Fase V & $\begin{array}{c}\text { IWA-V-1 } \\
\text { IWA-C-1 }\end{array}$ & $19<\mathrm{P}<75$ & 5 & $\mathrm{HC}+\mathrm{CO}<4,7$ & 0,3 & --- & 6 \\
\hline Fase V & $\begin{array}{c}\text { IWA-V-2 } \\
\text { IWA-C-2 }\end{array}$ & $75<\mathrm{P}<130$ & 5 & $\mathrm{HC}+\mathrm{NO} \times 5,4$ & 0,14 & -- & 6 \\
\hline
\end{tabular}

Las condiciones de contorno de nuestro problema, podrían enunciarse según esta frase: El mercado necesita más bancos de ensayo, para pruebas de emisiones en un menor plazo. La respuesta de AVL [3] -primera empresa a nivel mundial en bancos de ensayos de motores- es "fabricar" de forma modular el edificio de ensayos por completo, en vez de 
"construirlo" en casa del cliente. Un banco de ensayos de motor (véase Figura 2), consiste en una sala cerrada, aislada acústica y térmicamente dentro de la cual se prueba un motor de combustión. El motor se acopla al dinamómetro, que simula el par resistente. Para fijarlo al suelo, se tiene una bancada de acero. Puesto que el motor se encuentra desnudo, todos sus auxiliares - radiador, circuito de aceite, bomba combustible- han de ser simulados mediante equipos de acondicionamiento. La sala cuenta con una ventilación, para eliminar el calor del motor y una toma de aire fresco y otra de escape de humos. Estas salas tienen diferentes propósitos, según lo que se requiera ensayar. Uno de los más comunes, son ensayos de emisiones de gases contaminantes.



Figura 2: Vista general sala de ensayo

De todas las disciplinas que intervienen en este proyecto (obra civil, mecánica, electricidad...) el presente artículo se centra en las ramas relacionadas con la automatización.

La automatización, como pieza clave de la ingeniería de bancos de ensayo en contenedor, ha de cumplir con los plazos y calidades requeridas por el cliente

Del enunciado anterior se extraen los siguientes objetivos, de los que se ha partido en este artículo.

- Programación de PLCs. Desarrollo de código y depuración de un total de 5 PLCs de la gama S71200 de Siemens. Desarrollo de maniobras de funcionamiento, alarmas, parámetros de mantenimiento y controladores PID.

- Programación de sistema SCADA: Desarrollo gráfico y de interface de un total de 3 sistemas SCADAs para la visualización, registro de datos, alarmas, eventos y tareas de mantenimiento. La herramienta para este desarrollo ha sido el software AVL TFControl [3]. A mayores, para uno de los PLCs de la instalación, por tratarse de una máquina standard, se desarrollará un sistema HMI dedicado, basado en servidor web embebido en su PLC.
- Implementación de comunicaciones industriales. Programación de comunicaciones Profibus DP con sistema Maestro AVL PUMA [3]. Programación de comunicaciones S7 entre diferentes PLCs de la instalación, incluidos PLCs propios y de terceros. Se estandarizará la estructura de programación, para reducir plazos y costes.

- Puesta en marcha de la instalación: recepción de armarios eléctricos en taller. Pre puesta en marcha de las instalaciones en Austria y puesta en marcha final en Ankara, Turquía. Generación de certificados de prueba y protocolos de aceptación por el cliente.

- Solución de acceso remoto fiable y estándar dentro de la empresa: Estudio de mercado sobre las distintas soluciones de acceso remoto a PLCs. Comparativa entre solución de desarrollo propio y comercial. Implementación de software de acceso remoto basado en tecnología VPN

Para la presentación de los trabajos llevados a cabo en la consecución de los objetivos precedentes, el presente artículo se ha estructurado de la siguiente manera: en la sección 2 se hace una descripción de la instalación, presenta un layout de la instalación, así como los principales componentes que son directamente controlados por el sistema de automatización y o tienen interface con él; en la sección 3 se explican en las tareas de automatización los diferentes pasos realizados para automatizar la instalación descrita en el capítulo anterior. Esta sección a su vez se divide en varias subsecciones, según las diferentes disciplinas implicadas en el proyecto como son el desarrollo PLC, desarrollo SCADA y acceso remoto. En la sección 4 se presentan las conclusiones extraídas y se finaliza el trabajo con la lista de referencias utilizadas y citadas.

\section{DESCRIPCIÓN LA INSTALACION}

El edificio de ensayos (Figura 3) está construido dentro del recinto de la fábrica, como un edificio independiente. Se sitúa junta a la campa de acopio de unidades fabricadas. El proyecto completo, consiste en el suministro e instalación de los siguientes componentes:

- Edificio principal. Celdas de ensayo

- Contenedor de servicios. Grupos de bombeo, depósitos de inercia y compresor

- Enfriadora para producir agua fría a $2-7^{\circ} \mathrm{C}$

- Transformadores y armarios de distribución.

Los dos edificios cuyas instalaciones tenemos que automatizar son el edificio de ensayos (Figura 4) y el contenedor de servicios (Figura 5) 


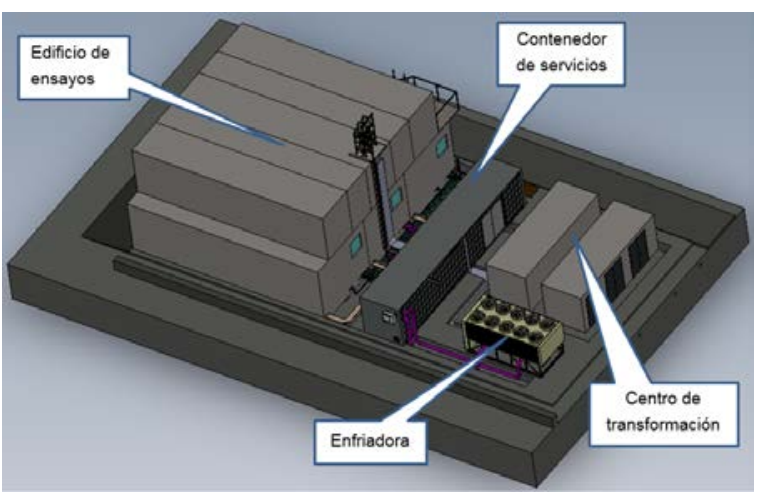

Figura 3: Vista general de la instalación

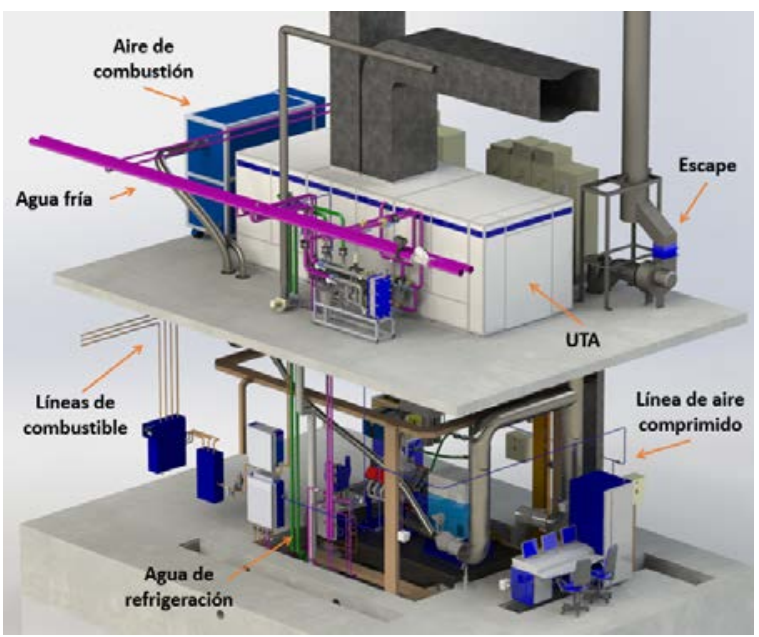

Figura 4: Edificio principal. Celda1.

La Figura 4 muestra los componentes del banco de ensayo y sus instalaciones asociadas. El edificio principal consta de dos celdas de ensayo. Las celdas se sitúan en la planta baja, y las instalaciones necesarias para cada una de ellas en la planta superior. En la planta superior se encuentran las unidades de ventilación, los equipos de aire de admisión y los extractores de escape. El edificio principal cuenta con dos salas de ensayo, separadas por una sala de equipos de emisiones y otra de almacenamiento de botellas de gases. En el edificio principal también se encuentran las salas de control.

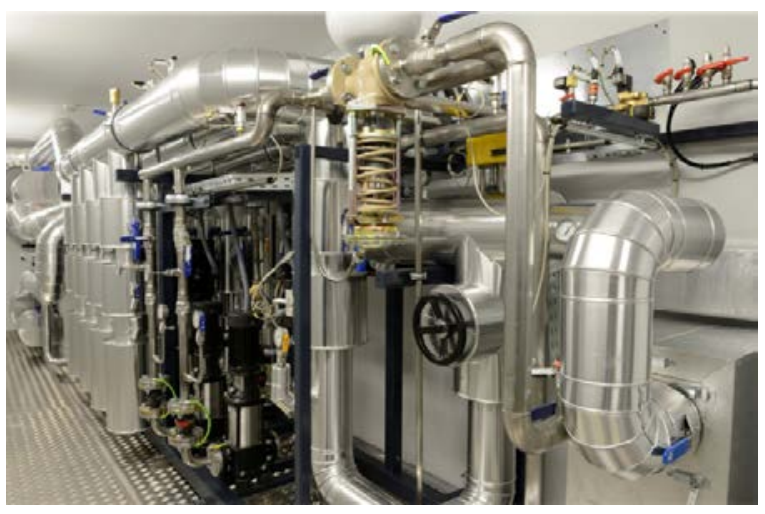

Figura 5: Contenedor de servicios
El contenedor de servicios, mostrado en la Figura 5 se encarga de proveer los diferentes fluidos y energías primarias al edificio principal.

- Agua $7 / 12^{\circ} \mathrm{C}$

- Agua $25^{\circ} \mathrm{C}$

- Combustible

- Aire comprimido.

Se tiene un circuito de agua helada que envía agua a $7 / 12^{\circ} \mathrm{C}$ al edificio principal. Los consumidores de esta agua en el edificio principal son las unidades de ventilación y aire de admisión, los acondicionadores de combustible y los equipos de refrigeración de intercooler. El principio de funcionamiento del sistema es el siguiente: Cuando el sistema se enciende, las bombas de velocidad constante arrancan inmediatamente, enviando el agua hacia la planta enfriadora. Después de la enfriadora, nos encontramos con los tanques de inercia, donde el agua se almacena para aumentar la disponibilidad del sistema y se reducen las posibles fluctuaciones de temperatura. Puesto que puede que no todos los consumidores de agua estén demandando a la vez, el grupo de bombeo variable, ajusta su velocidad utilizando un controlador PID en función de la presión diferencial entre el punto de impulsión y retorno hacia las celdas.

Del mismo modo, se tiene un circuito de agua fría a $\sim 25^{\circ} \mathrm{C}$. El principio de funcionamiento del sistema es similar al anterior. La generación de frío se realiza mediante un intercambiador de calor contra el circuito de agua helada. La temperatura de salida de este intercambiador se controla realizando un PID contra una válvula de 3 vías situada en el primario del intercambiador, en el circuito de agua helada. La bomba en esta aplicación es de velocidad constante y la regulación de caudal se realiza utilizando una válvula reguladora de presión en impulsión. La regulación es puramente mecánica, no interviene el sistema de automatización. El principal consumidor de este circuito es el propio motor que se está probando en la celda de ensayo.

El contenedor de servicios también es responsable del suministro de combustible a las celdas. Para mantener constante la presión a la entrada de los equipos de medida y acondicionamiento de combustibles, el combustible es bombeado a unos depósitos de 60 litros - uno para cada tipo de combustible- situados en la terraza del edificio principal. Estos pequeños depósitos de combustible se conocen como jornaleros. El funcionamiento del sistema es sencillo. En cada una de las celdas, el utilizador puede seleccionar combustible del tipo $1 \mathrm{o}$ de tipo 2. Esto abre la válvula de solenoide del combustible correspondiente. El nivel del tanque está monitorizado por un sensor de flotador. En el momento que se detecta nivel bajo, la válvula de 
entrada se abre hasta que el nivel vuelve a ser alto. Existe una alarma por nivel demasiado alto, que cierra automáticamente la entrada de combustible. En el caso del combustible de los barriles, el relleno del tanque es manual. El operador ha de activar una bomba para poder volver a llenar el tanque superior. En cuanto al aire comprimido, se trata de una red de 6bar. Los únicos elementos de supervisión o control que tiene el sistema de automatización en este circuito, son una electroválvula de corte y un presostato. La válvula de corte cerrará cuando se produzca algún evento de seguridad en el interior de la celda (paro de emergencia, incendio...)

\section{TAREAS DE AUTOMATIZACIÓN}

Una vez entendida la instalación se ha realizado una selección de sensores - de manera conjunta con los diseñadores de fluidos- para controlar el proceso. Se han empleado sensores para la medida de temperaturas TN2531 [5]. Los sensores de presión utilizados son PN2024 [6] en el caso de medidas continuas, y PK5524 [7] en el caso de presostatos. Se han empleado detectores de flujo modelo SI6800 [8] y detectores de nivel KG8008 [9]. Los detectores de nivel empleados para combustibles, han de ser intrisnecamente seguros. Por ello se ha recurrido al modelo KSB KUEBLER 1003 [10]. En cuanto a los actuadores, las válvulas todo o nada son Genebre modelo 5528 [11], y las proporcionales son Sauter modelo AVM234S [12]. Los variadores de velocidad empleados para el control de bombas son Siemens Sinamics G120C. Una vez seleccionados los sensores y conectados a los armarios de control, se dispone de la lista entradas y salidas, punto de partida para las tareas de automatización y desarrollo HMI. Se han programado los elementos de la Tabla 2

Tabla 2: elementos de automatización.

\begin{tabular}{|l|l|}
\hline Elemento & Descripción \\
\hline LVDC00 & PLC contenedor de servicios \\
\hline LVDC01 & PLC control celda 1 \\
\hline LVDC02 & PLC control celda 2 \\
\hline LVDC03 & PLC control sala emisiones \\
\hline SCADA00 & SCADA monitorización servicios \\
\hline SCADA01 & SCADA monitorización celda 1 \\
\hline SCADA02 & SCADA monitorización celda 2 \\
\hline
\end{tabular}

\subsection{DESARROLLO PLC}

La solución de automatización se ha realizado empleando PLCs Siemens S7-1200. Se ha situado un PLC dentro de cada uno de los armarios eléctricos, en cada uno de los contendores según Figura 6 y Figura 7. Disponer de un PLC en cada uno de los contenedores, nos permite probarlos por separado antes de la instalación final.

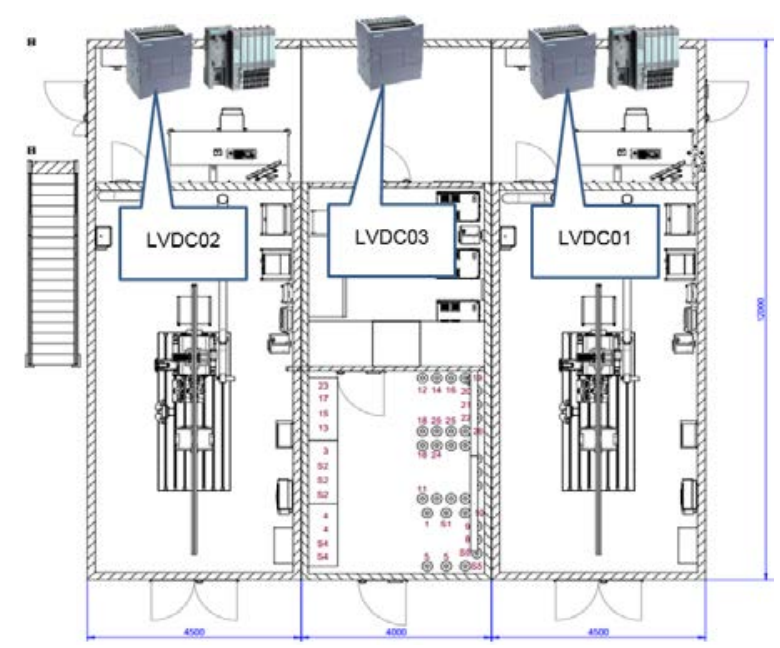

Figura 6: Posición de PLCs. Edificio principal.



Figura 7: Posición de PLCs. Contenedor de servicios

Los PLCs se nombran de la misma manera que el armario eléctrico que los contiene. Las tareas que realizan los PLCs del edificio principal, son la vigilancia de elementos de los propios armarios eléctricos, como los auxiliares de automáticos, guardamotores y contactores. Del mismo modo, controla los principales servicios de la celda, como la unidad de ventilación, válvulas de acometida, enchufes y tomas pilotas. El PLC del contenedor de servicios realiza todas las funciones anteriores y a mayores es el encargado de la regulación de los circuitos de agua fría y agua helada.

La Figura 8 muestra el sinóptico de proceso del control de temperatura del agua fría y sobre él el flujo de información hacia y desde el controlador PID. La variable que se pretende controlar, es la temperatura de impulsión del agua fría. Para ello, el valor medido por el PID es la sonda de temperatura que se encuentra en la aspiración de las bombas. El valor que se pretende conseguir en esa sonda es de aproximadamente $30^{\circ} \mathrm{C}$. Para ello, se cuenta con una válvula de control de tres vías. Esta válvula, está instalada en configuración mezcladora, a la salida del intercambiador de calor, balancea el caudal de agua helada que trasiega por el primario del intercambiador de calor. 


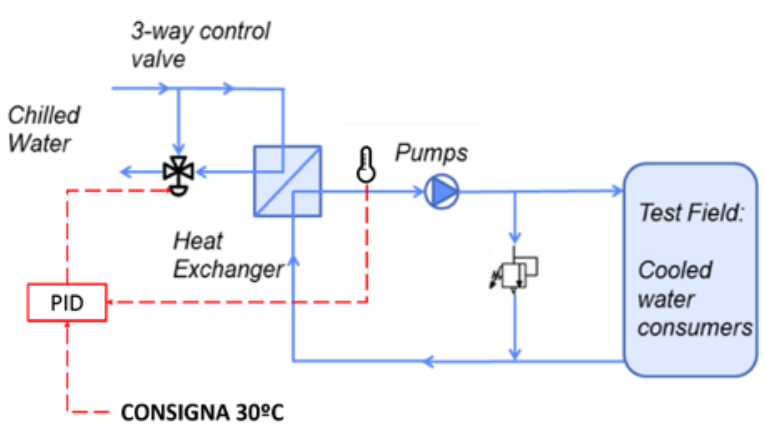

Figura 8: Esquema circuito agua fría

De este modo se controla la potencia transmitida por el intercambiador de calor y por consiguiente la temperatura del agua a su salida. La precisión de la temperatura que se quiere conseguir en este control no es muy demandante. Un control de $+/-5^{\circ} \mathrm{C}$ suele considerarse aceptable, ya que el principal consumidor de este tipo de agua es la refrigeración del bloque motor. Este control no se realiza directamente utilizando el agua de este circuito, si no que existe un equipo de acondicionamiento que cuenta con un intercambiador de calor que es el encargado de conseguir una precisión de regulación mayor en el circuito del motor a ensayar.

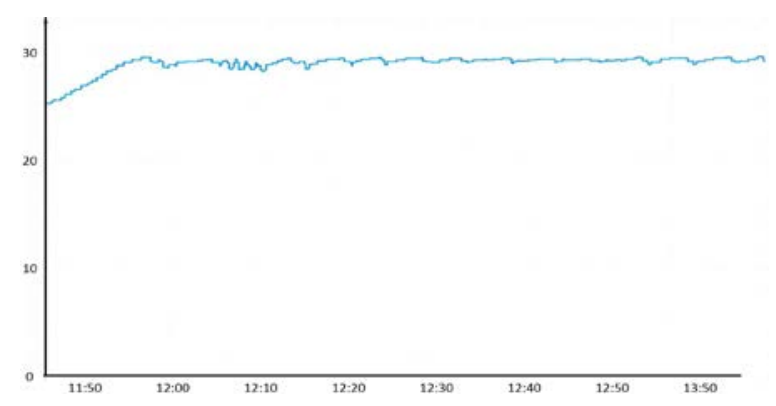

Figura 9: Control temperatura de agua fría

La Figura 9 muestra el gráfico de aceptación por parte del cliente final de la regulación del control de temperatura. Tanto la potencia de refrigeración como su control de temperatura son adecuados para el proceso. La oscilación que se observa durante los primeros ciclos tras el arranque no es un problema. Parece que puede ser debida al agua acumulado en el tramo de bypass de la instalación tras el primer arranque. En el momento que todo el agua empieza a circular y las temperaturas en los distintos puntos se estabilizan, la actuación de la válvula se estabiliza y el control de temperatura también.

La Figura 10 muestra el sinóptico de proceso del control de presión del agua helada y sobre él el flujo de información hacia y desde el controlador PID. La variable que se pretende controlar, es la diferencia de presión de impulsión y retorno del agua helada. Para ello, el valor medido por el PID es la resta de los valores medidos por los sensores de presión de impulsión y retorno.

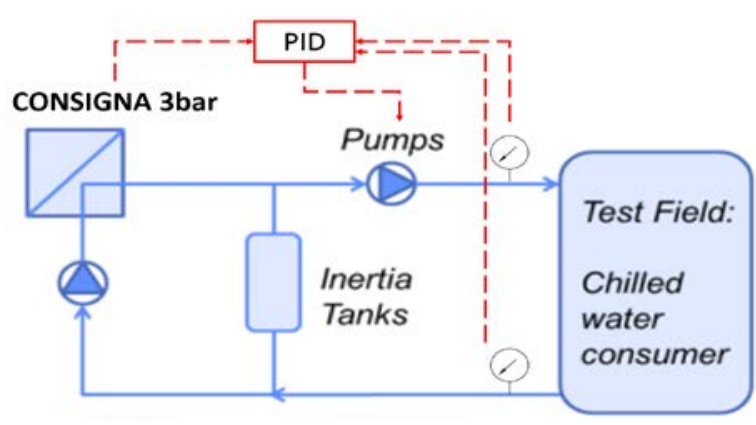

Figura 10: Esquema circuito agua helada

El valor que se pretende conseguir en esa diferencia de medidas es de 3 bar. Para ello, se cuenta con dos bombas, cuyos motores están comandados por sendos variadores de frecuencia. Conforme se abren los diferentes consumidores de agua helada en el lado de las celdas, la resistencia hidráulica del circuito disminuye. El caudal que han de enviar las bombas hacia el proceso es mayor, para satisfacer la demanda de los nuevos consumidores. Para mantener el caudal que requiere cada uno de estos consumidores, es necesario ajustar la presión de red. Para aumentar la presión, se aumenta la velocidad de giro de las bombas mediante variadores de velocidad.

El requerimiento de precisión en este control es mayor que en el control de temperatura. Los consumidores de este circuito están diseñados asumiendo una determinada presión de red. En el caso de que la presión de red salga del rango esperado, estos consumidores (acondicionador, de combustible, intercooler, ventilación...) dispararán alarmas y no funcionarán. No existe un valor requerido por el cliente. Se asumirá como valor límite, una diferencia de presión de $+/-0.2$ bar.

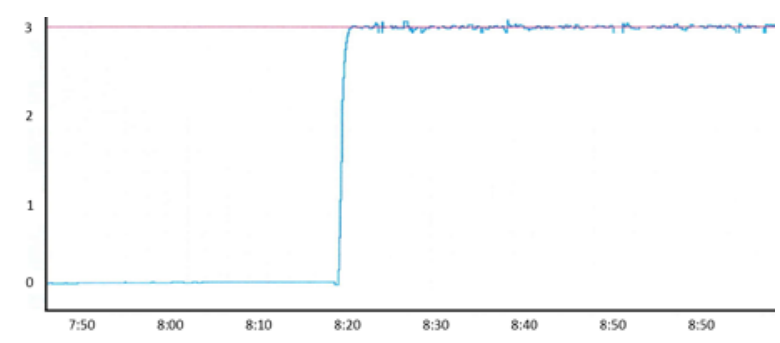

Figura 11: Control presión de agua helada

La Figura 11 muestra el gráfico de aceptación por parte del cliente final de la regulación del control de presión. Puesto que el control de presión es un control intermedio - la magnitud que tienen que controlar los acondicionadores es la temperatura- es fundamental que este control cumpla dos requisitos básicos: ha de ser más rápido en orden de magnitud que el control de temperatura (si las constantes de tiempo fuesen similares, ambos controladores se podrían acoplar); también ha de ser más preciso. Una oscilación en el control de presión, variará el flujo a 
través de los intercambiadores de calor, ocasionando oscilaciones en el control de temperatura.

Una vez descritas las funciones de cada uno de los PLCs por separado, la Figura 12 lo ilustra.

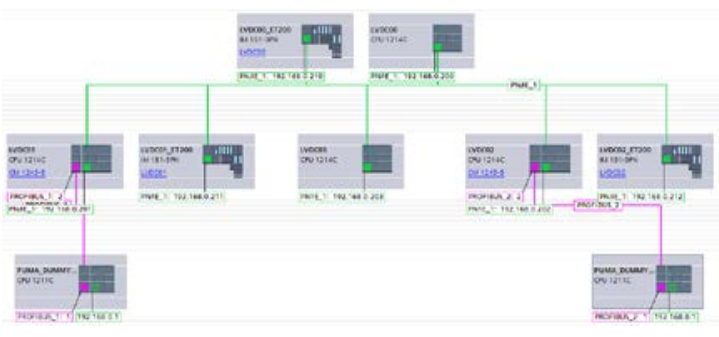

Figura 12: Estructura de red

Los PLCs de celda comunican con el PC industrial AVL Puma a través del protocolo Profibus DP, actuando como esclavos Profibus. Para ello se emplea el módulo CM1242-5 de siemens. Del mismo modo, cada uno de los PLCs de celda, comunica con la ventilación de la celda correspondiente utilizando el protocolo S7, basado en Ethernet. Los PLCs de la ventilación son S7-300 y han sido programados por un tercero. Cada uno de los PLCs de celda comunica con el PLC del contenedor de servicios mediante otra comunicación S7.

\subsection{DESARROLLO SCADA}

Se ha desarrollado un sistema SCADA para el control de las instalaciones, tanto a nivel de celda como a nivel de contenedor de servicios. En cuanto al hardware, se han instalado 3 PCs físicos, formato all-in-one. Uno para cada celda de ensayo y otro para el contenedor de servicios. A nivel software, la herramienta empleada es el software AVL TFControl [3].

Es un sistema SCADA desarrollado por AVL, enfocado a instalaciones de ensayos de motores. El desarrollo y parametrización de las pantallas de la instalación se desarrolla en Adobe Flash ${ }^{\circledR}$. El SCADA AVL TFControl [3], tiene una interfaz gráfica interactiva, en la que se muestra el sinóptico del proceso y los botones para interactuar con él. Del mismo modo, cuenta con una base de datos MySQL en la que se almacenan un log de las diferentes variables del proceso. Estas variables se pueden graficar utilizando la herramienta RRDD tools. En la BBDD también se guardan tablas de alarmas, y eventos, con los diferentes mensajes de alarma y error que producen los PLCs.

El SCADA AVL TFControl también cuenta con un sistema de control de usuarios, que permite configurar el grado de acceso de cada uno de los usuarios registrados. Se tiene también un sistema de alarmas remotas, que permite enviar mensajes SMS o de correo electrónico configurables. Las pantallas que componen el SCADA de cada una de las celdas se pueden ver en las Figuras 13 a 17.

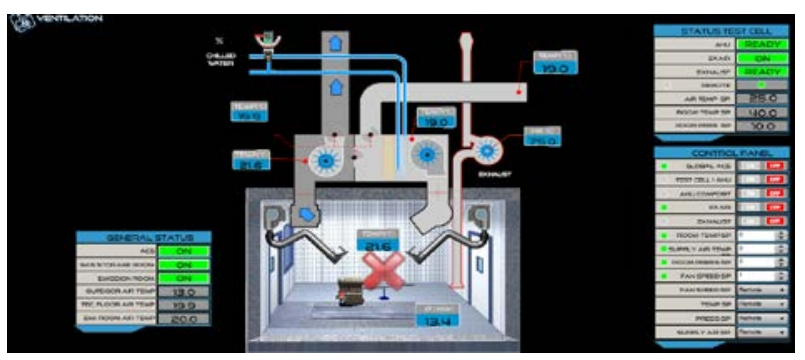

Figura 13: SCADA celda. Ventilación

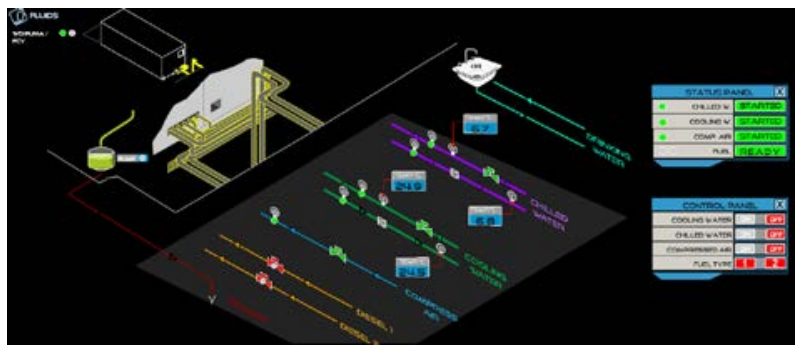

Figura 14: SCADA celda: Fluidos

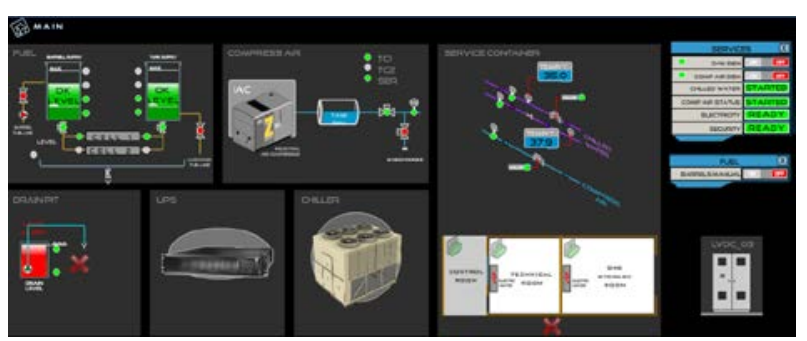

Figura 15: SCADA servicios. Principal

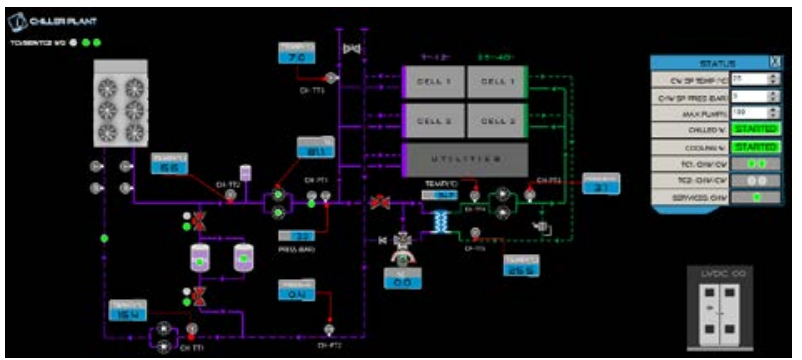

Figura 16: SCADA servicios. Agua fría y helada.

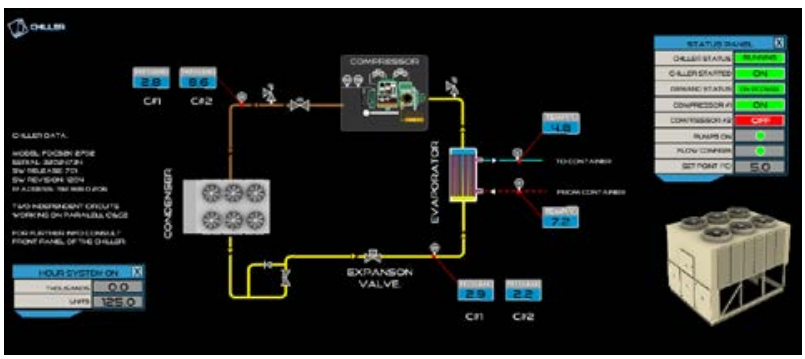

Figura 17: SCADA servicios. Enfriadora

Para el equipo AVL Battbox, situado en la celda de ensayo, se ha realizado a mayores un HMI basado en HTML, Figura 18, utilizando la funcionalidad de servidor web que tienen embebida los PLCs S7-1200. 


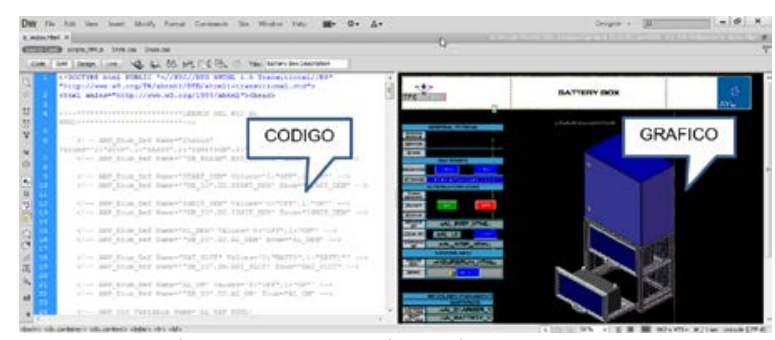

Figura 18: HMI basado en HTML

Estos HMIs se programan introduciendo un código desarrollado en el encabezamiento de la página web. Dicho código, que tiene la apariencia de un comentario HTML, es interpretado por el servidor web del PLC, sustituyendo los valores codificados por valores de variables del PLC, enlazando así la memoria del PLC con la visualización en un navegador.

\subsection{ACCESO REMOTO}

A día de hoy internet es un elemento común en las aplicaciones de automatización industrial. Ya sea para recopilar datos de funcionamiento de las instalaciones, para el aviso de alarmas por mail o para tele asistencia, el número de instalaciones que incorporan un acceso a internet es cada vez mayor.

La tecnología elegida para realizar el acceso remoto es la red VPN. Una red VPN (virtual private network) es una extensión de una red de área local a través de otra red intermedia, como puede ser internet. La utilización de redes VPN es habitual en el campo de la informática, para soluciones de teletrabajo o interconexión entre varias oficinas de una misma empresa. Existen varios tipos de redes VPN, como pueden ser PSEC o PPTP. Nos vamos a centrar en el protocolo Open VPN, por ser de código abierto y contar con abundante documentación.

La arquitectura de red es relativamente sencilla. Se establece un servidor VPN, que está a la escucha esperando recibir conexiones por parte de los clientes. A nivel de seguridad, cada uno de los clientes, que conoce la dirección del servidor, le envía una petición de conexión. Para que un cliente se pueda conectar a este servidor, ambos tienen que conocer la clave precompartida de hasta 256bit y un certificado, que ha generado el servidor previamente para cada uno de los clientes. Estos certificados tienen una validez de 10 años en el servidor y han de guardarse en ambos dispositivos, cliente y servidor.

A nivel de enrutamiento, una vez que cada cliente y servidor cuenta con las condiciones de seguridad necesarias, se deben crear los adaptadores de red VPN. La realización práctica del túnel VPN consiste en la creación de un adaptador de red (igual que nuestra antena wifi o el conector de Ethernet) virtual llamado driver tap. En el servidor, se realizará una ruta que redireccione el tráfico recibido por los adaptadores virtuales.

Se necesita definir a nivel hardware y software cada uno de los siguientes componentes.

Cliente OVPN en el lado PLC: ha de ser un dispositivo pequeño, que se pueda instalar dentro de un armario eléctrico. Ha de tener varias posibilidades de conexión a internet (utilizando tarjeta sim, wifi o una conexión Ethernet). Ha de ser sencillo de configurar y económico.

Cliente OVPN en el lado del usuario: lo más común es un PC con Windows, donde se tenga instalado TIA Portal [13] y el SCADA de la instalación. Se necesita un interface sencillo para elegir la instalacion con la que nos queremos conectar. Control de usuarios y acceso por contraseña.

Servidor OVPN: ha de ser accesible desde cualquier lugar del mundo (ip fija) tiene que tener alta disponibilidad y baja tasa de fallos. No debe requerir un gran esfuerzo de mantenimiento a nivel software.

Parece claro que la mejor opción para el servidor OVPN es un ordenador "en la nube" frente a un servidor propio. De esta manera el servicio, la velocidad y la disponibilidad mundial están garantizados. Para el cliente en el lado PLC, se barajó la posibilidad -incluso se ha llegado a implementar con éxito- utilizar el PLC del SCADA como cliente VPN y enrutar todo el tráfico de la LAN a su través. Esta opción se descartó, ya que el ordenador del SCADA es un PC que puede estar apagado, por ejemplo por ser la causa de la avería. No parecía la mejor opción para implementar el cliente VPN

La siguiente opción considerada, fueron los routers de altas prestaciones y low cost de la marca Mikrotik ${ }^{\circledR}$. Estos routers son $100 \%$ configurables. Tienen implementado un sistema operativo basado en Linux llamado routerboard y nos permiten montar en un dispositivo que cabe en la palma de la mano un servidor web, un firewall, un cliente VPN... Esta opción cumple todas las condiciones que habíamos enumerado, salvo la sencillez. La configuración de este tipo de routers exige un perfil de usuario informático avanzado. La idea es que el router VPN, entre otras tareas, ayude a los técnicos de puesta en marcha, no que les sea un problema añadido. Aunque esta solución también era funcional, no se llegó a implementar por ser demasiado compleja.

La solución elegida finalmente (Figura 19) fue la proporcionada por el fabricante de routers industriales Ewon ${ }^{\circledR}$, que provee un una solución incluyendo los dos clientes VPN y aloja el servidor VPN en la nube. 


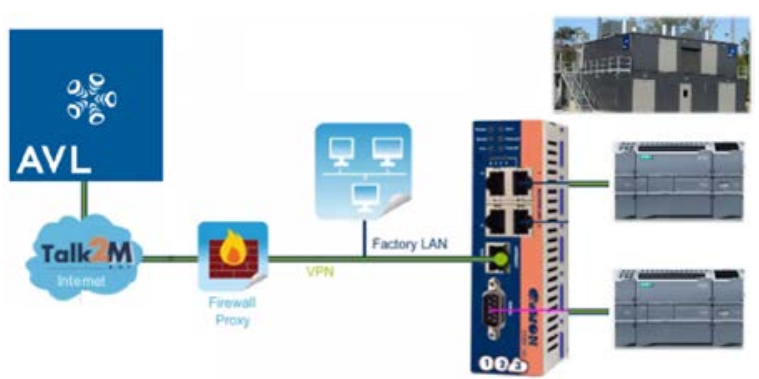

Figura 19: Arquitectura del acceso remoto

\section{CONCLUSIONES}

Tras la finalización del presente trabajo, se pueden extraer las siguientes conclusiones.

- Se ha desarrollado el código de un total de 5 PLCs de la gama S7-1200 de Siemens. Se han programado las maniobras de funcionamiento, alarmas, parámetros de mantenimiento $\mathrm{y}$ controladores PID. Se han implementado las comunicaciones industriales utilizando buses de campo entre los diferentes PLCs así como el ordenador industrial AVL Puma.

- Se ha programado un sistema SCADA realizando el desarrollo gráfico y de interface de un total de 3 sistemas SCADAs para la visualización, registro de datos, alarmas, eventos y tareas de mantenimiento. La herramienta para este desarrollo ha sido el software AVL TFControl. Se han comprado e instalado 3 terminales de acceso a este SCADA, basados en ordenadores táctiles. A mayores, para uno de los PLCs de la instalación, por tratarse de una máquina standard, se desarrolló un sistema HMI dedicado, basado en servidor web embebido en su PLC.

- Se ha hecho la puesta en marcha de la instalación recepcionado los armarios eléctricos en taller, haciendo prepuesta marcha en Austria, y finalizando con la puesta en marcha final en Ankara, Turquía, generando los certificados de prueba y protocolos de aceptación por el cliente.

- Se ha realizado una solución de acceso remoto fiable y estándar dentro de la empresa junto con el estudio de mercado sobre las distintas soluciones de acceso remoto a PLCs y comparativa entre solución de desarrollo propio y comercial. Implementando el software de acceso remoto basado en tecnología VPN.

- Se ha establecido un estándar en la forma de programación dado que se tiene un número importante de PLCs a programar, y que los mismos han de ser editados por diferentes programadores y técnicos de puesta en marcha. Para ello se han desarrollado unas directrices y normas de programación que fueron aplicadas en el presente proyecto de tal suerte que todos los PLCs se han programado siguiendo estas normas de programación, que han acabado convirtiéndose en el estándar de programación de AVL.

\section{Referencias}

[1] B. Morey, «Volvo's Rapid Strategy aims at 20month vehicle development,» $S A E, 2015$.

[2] EARTH POLICY INSTITUTE, «Grain harvest,» Earth policy institute, 24 Mayo 2017. [En línea]. Available: http://www.earthpolicy.org/indicators/C54/grain_2013. (Último acceso: 05 Mayo 2017).

[3] AVL, «AVL,» AVL, https://www.avl.com/home (Último acceso: 24 Mayo 2017).

[4] «REGLAMENTO (UE) 2016/1628 DEL PARLAMENTO EUROPEO Y DEL CONSEJO del 14 de septiembre 2016,» https://www.boe.es/doue/2016/252/L0005300117.pdf. (Último acceso14 septiembre 2016).

[5] IFM, «IFM -TN2531,» http://www.ifm.com/products/es/ds/TN2531.ht ml. (Último acceso:13 April 2017).

[6] IFM electronic, «IFM-PK5524,» http://www.ifm.com/products/es/ds/PK5524.ht ml. (Último acceso: 13 Abril 2017).

[7] IFM electronic, «TFM-PN2024,» http://www.ifm.com/products/es/ds/PN2024.ht ml. (Último acceso: 13 aBRIL 2017).

[8] IFM electronic, «IFM-SI6800,» http://www.ifm.com/products/es/ds/SI6800.html (Último acceso: 13 Abril 2017).

[9] IFM electronic, «IFM-KG0008,». http://www.ifm.com/products/es/ds/KG0008.ht ml. (Ultimo acceso:13 Abril 2017).

[10] KSR KUEBLER, «KSR KUEBLER 1003,» http://www.processpartner.no/files/processpartn er.no/Documents/Instrumentering/Niv\%C3\%A5 Niv\%C3\%A5brytere/KSR/10034\%20Float $\% 20$ switches.pdf. (Último acceso: 13 Abril 2017).

[11] GENEBRE, "GENEBRE-5008,» http://www.genebre.es/download/file/ZG9jdW1 lbnRvcy9vdHJvcy81ODAwX2duLnBkZg== (Último acceso: 13 Abril 2017)

[12] SAUTER, «SAUTER AVM 234S,» http://www.sautercontrols.com/uploads/tx cabagpdm/873116.pdf. (Último acceso: 13 Abril 2017).

[13] SIEMENS, «SIEMENS AUTOMATION SUPPORT,» SIEMENS, https://support.industry.siemens.com/cs/search? search=siemens $\% 20$ tia $\% 20$ portal. (Último acceso: 24 Mayo 2017) 